\title{
Metal incorporation into nanoporous carbon
}

\author{
S. J. Henley ${ }^{1}$, N. E. P. Woolger ${ }^{1}$, J. D. Carey $^{1}$, S. R. P. Silva ${ }^{1}$, G. M. Fuge ${ }^{2}$ and M. N. R. Ashfold ${ }^{2}$ \\ ${ }^{1}$ Advanced Technology Institute, School of Electronics and Physical Sciences, \\ University of Surrey, Guildford, GU2 7XH, U.K. \\ ${ }^{2}$ School of Chemistry, University of Bristol, Bristol, BS8 1TS, U.K.
}

\begin{abstract}
Nanoporous carbon films were deposited by $248 \mathrm{~nm}$ pulsed laser ablation of a graphite target in different background pressures of argon $\left(\mathrm{P}_{\mathrm{Ar}}\right)$. The morphology changed from smooth, high-density amorphous carbon films at $\mathrm{P}_{\mathrm{Ar}}=20 \mathrm{mTorr}$ to ultra-low density nanoporous material at $\mathrm{P}_{\mathrm{Ar}}=380 \mathrm{mTorr}$. Subsequently, the nanostructural, chemical and electrical properties of metal containing nanoporous carbon samples were investigated by ablating graphite targets containing known contents of $\mathrm{Ni}$ and $\mathrm{Co}$. We demonstrate how the ablation plume dynamics affect both the nanostructure of the material and the incorporation of metal atoms. The suitability of these functionalised ultra-low density materials for gas sensing applications is discussed.
\end{abstract}

\section{INTRODUCTION}

The presence of ambient gas can have a dramatic effect on the expansion dynamics of laser-ablated plasmas [1]. Nanosecond pulsed laser ablation (PLA) of graphite results in the deposition of quite different carbonaceous structures depending on the pressure of ambient gas in the chamber [2,3]. PLA of graphite in vacuum is typically used to produce smooth, hard, high-density amorphous carbon (a-C) films with high $\mathrm{sp}^{3}$ bonded $\mathrm{C}$ content (up to $90 \%$ ) $[4,5]$. The introduction of a low pressure of inert gas into the growth chamber reduces the kinetic energy of the growth species due to the increased collision frequency. At higher pressures plume confinement, and plume heating occurs, which leads to enhanced excitation/ionisation at the plume front. Compared with growth in vacuum, these conditions have been shown to yield lower density nanoporous carbon (NPC) films [3,6], which can exhibit a range of useful properties including low thresholds for electron emission [7] and high porosities [3] suggesting gas sensor applications [8]. Recent work on high repetition rate laser ablation of carbon in background pressures of argon has shown that these conditions can deposit carbon nanofoam [9].

However, porous carbon samples have been found typically to have very high resistivities $[8,9]$ of the order of $10^{7}-10^{9} \Omega \mathrm{cm}$, limiting their usefulness in electronic devices applications. One possible technique for improving the conductivity of these films is by incorporating metal atoms during the growth. The doping of $\mathrm{a}-\mathrm{C}$ thin films by incorporation of metals has been attempted and it was found that the mechanical, tribological, and electrical properties are all strongly influenced by the metal content in the films [10, 11]. For example, electrical conductivity could be increased many orders of magnitude by varying the metal content [11-13]. In this paper we investigate the incorporation of metal into the NPC films deposited by PLA of graphite. We examine the growth mechanisms, and determine how the ambient pressure during the deposition affects the metal content and the electrical properties.

\section{EXPERIMENTAL DETAILS}

Nanoporous carbon films (NPC) were deposited by PLA of a $99.999 \%$ pure pyrolytic graphite target (Kurt J. Lesker) using a Lambda-Physik LPX 210i excimer laser operating at $248 \mathrm{~nm}$. The laser pulse was focused onto the rotating target, producing a fluence of $\sim 6 \mathrm{~J} / \mathrm{cm}^{2}$. The growth chamber was evacuated using a turbomolecular pump to $9 \times 10^{-8}$ Torr, then back-filled with argon to the required pressure. Films were deposited onto $\mathrm{Si}$ and glass substrates at a range of different background pressures of argon $\left(\mathrm{P}_{\mathrm{Ar}}\right)$ from 5 mTorr to 340 mTorr. The target to substrate distance was fixed at $6 \mathrm{~cm}$. Between 2000 and 5000 laser shots were used, depending on the background pressure, as the film thickness was affected by the $\mathrm{P}_{\mathrm{Ar}}$ in the chamber. The number of laser shots was varied to keep the final thickness of the films within a fixed range. For the metal 
containing NPC films the pure graphite target was substituted for either a mixed target of graphite with $20 \%$ (by number of atoms) of the required metal, or by a composite target of graphite and a high purity metal foil. In this second arrangement, fast rotation of the composite target allowed periodic ablation of the graphite and the metal foil to produce a mixed material. The morphology of the films was investigated in a Hitachi S4000 field emission gun scanning electron microscope (SEM) operating at $20 \mathrm{kV}$ and energy dispersive X-ray analysis (EDX) in a Philips XL30 SEM provided measurements of the chemical composition of the films. The nanostructure of these materials was examined in a Philip CM200 transmission electron microscope (TEM). The ablation plume imaging and spectroscopy part of this experiment was carried out in a separate dedicated apparatus which is detailed elsewhere [14].

\section{RESULTS AND DISCUSSION}

Before discussing the incorporation of metal into NPC, we must first investigate the growth mechanisms without the presence of metal. Figure 1 shows SEM images of a range of samples deposited at different $\mathrm{P}_{\mathrm{Ar}}$ by ablating the pure graphite target. At the lowest $\mathrm{P}_{\mathrm{Ar}}$ used $(5 \mathrm{mTorr})$ the films deposited were hard, scratch resistant and demonstrated a smooth morphology (see figure 1(a)). As $\mathrm{P}_{\mathrm{Ar}}$ was increased to $40 \mathrm{mTorr}$ (figure 1(b)) the films became much rougher. The films deposited at 100 mTorr could be easily scratched off with a pair of tweezers, indicating a much lower hardness. By 340 mTorr (see figure 1e) the film morphology had changed from nanoscale roughness to more fractal-like growth, characteristic of diffusion-limited aggregation of clusters.

In order to determine how $\mathrm{P}_{\mathrm{Ar}}$ affected the structure of our samples, the velocity of a selection of the $\mathrm{C}$ species in the ablation plume was estimated from series of images of the optical emission from $\mathrm{C}^{+}$species using a technique discussed elsewhere [15]. Estimates of the mean velocities $\langle\mathrm{v}\rangle$ of the $\mathrm{C}^{+}$species in the ablation plume were derived by analysing the emission intensity normal to the target of CCD plume images filtered to only show the $\mathrm{C}^{+}$emission at $427 \mathrm{~nm}$. Figure 2 shows the way in which the derived peak in the profiles $\mathrm{x}_{\text {peak }}$ varies with $\mathrm{t}$, as a function of $\mathrm{P}_{\mathrm{Ar}}$. Figure 2 illustrates the good linear relationship between $\mathrm{x}_{\text {peak }}$ and time $t$ observed for $248 \mathrm{~nm}$ PLA of graphite, in vacuum. The gradient of this plot gives a propagation velocity $<v>$ of $41.5 \pm 0.1 \mathrm{~km} / \mathrm{s}$ for the $\mathrm{C}^{+}$species. This is equivalent to a $\mathrm{C}$ atom kinetic energy of $\sim 107 \mathrm{eV}$, and a-C films grown from ablation plumes containing $\mathrm{C}$ cations with energies in this range typically have a high $(\sim 80 \%)$ fraction of $\mathrm{sp}^{3}$ bonding [16]. Collisional contributions would be expected to increase, and the mean propagation velocity to slow, with increasing $\mathrm{P}_{\mathrm{Ar}}$. Consistent with such expectations we see that, for $\mathrm{P}_{\mathrm{Ar}}$ $>68$ mTorr the data can be reproduced well by a simple function of the form $\mathrm{x}_{\text {peak }} \propto t^{\alpha}$. Such an expression was introduced to model the expansion of a spherical blast-wave [17] - wherein the exponent $\alpha$ takes a limiting value of $\sim 0.4$. This implies a deceleration of the emitting species.

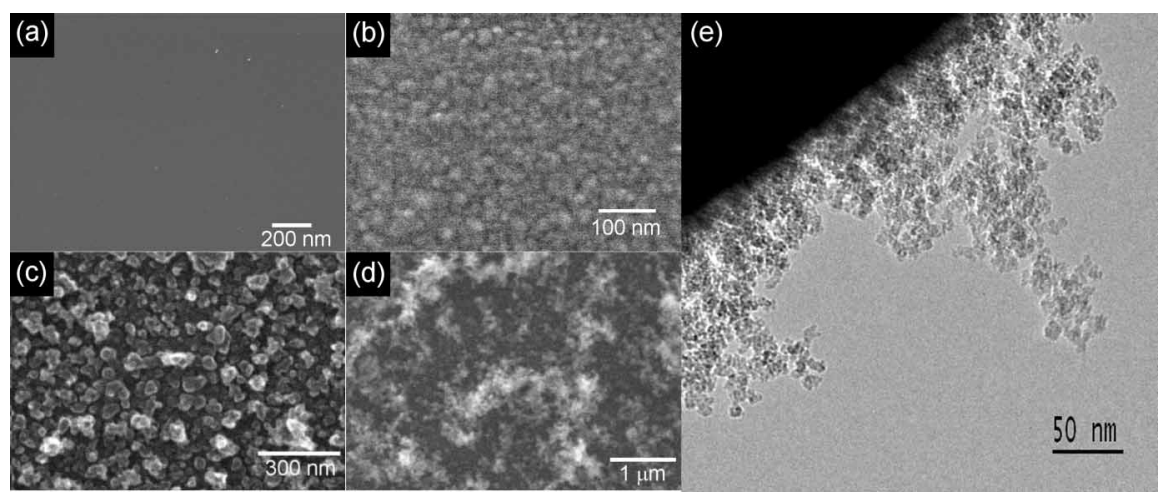

FIG. 1: SEM images of a range of samples deposited at different $P_{\text {Ar. }}$. (a) 5 mTorr, (b) 40 mTorr, (c) 100 mTorr and (d) 340 mTorr. Note the different scale bars on the images. (e) TEM image of the sample deposited at 340 mTorr. 


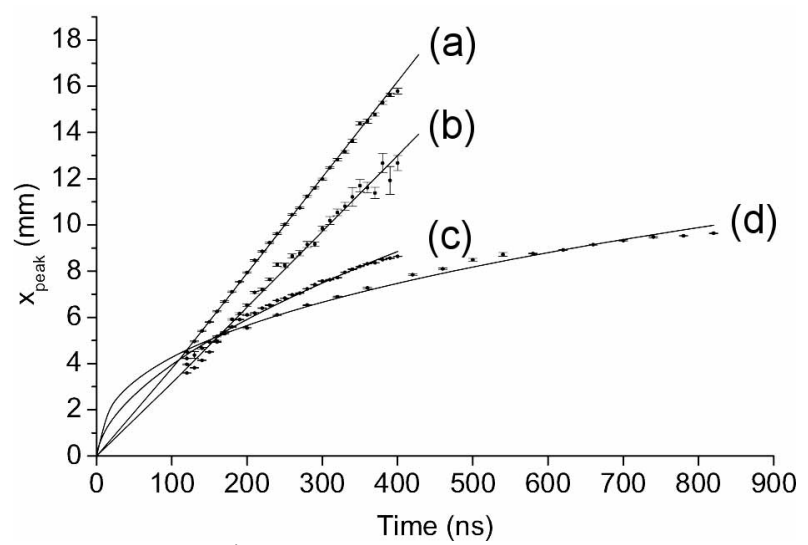

FIG. 2: Plots of xpeak versus time for the $\mathrm{C}^{+}$emissions following nanosecond PLA of graphite in background pressures of $\mathrm{P}_{\mathrm{Ar}}=$ (a) 0 mTorr (b) 20 mTorr, (c) $68 \mathrm{mTorr}$, and (d) 154 mTorr.

The rate of decrease in the deduced velocity with increasing $\mathrm{P}_{\mathrm{Ar}}$ and/or $t$ might suggest that the ablated material would not reach the substrate. However, the velocity we derive at high $\mathrm{P}_{\mathrm{Ar}}$ is really the propagation velocity of the $\mathrm{C}^{+}$ions within the shock wave formed as the plume expands into the background gas. Once the highly energetic ablated atomic material has been slowed by collisions, the formation of carbon clusters becomes a possibility. The size and bonding configuration within these clusters is then controlled by the plume temperature and density of carbon atoms [9]. From figure 1e we can see that the nanostructure of the films deposited at the higher background pressures appears to be consistent with growth by diffusion limited aggregation of clusters formed in the gas phase as the structure appears fractal-like. Over the pressure range studied the main growth species changes from high-energy carbon atoms and ions at low pressures, to slowly diffusing carbon clusters at higher pressures.

With the understanding of the growth mechanisms of carbon films in the argon pressure range studied, we then investigate how the incorporation of metal into the films is affected by $\mathrm{P}_{\mathrm{Ar}}$. To incorporate metal into the films, two different ablation configurations were investigated; a mixed target of graphite with $20 \%$ (by number of atoms) of the required metal and a composite target of graphite and a high purity metal foil. In this second arrangement, fast rotation of the composite target allowed periodic ablation of the graphite and the metal foil to produce a mixed material. The two metals investigated were $\mathrm{Ni}$ and $\mathrm{Co}$, as the incorporation of these elements has been shown to increase the electrical conductivity of a-C [12,13]. Figure 3 shows a series of SEM images of NPC:Ni samples deposited at different $P_{A r}$, by ablating the composite target. In vacuum (see figure 3a) the surface morphology observed is rougher than that observed for the metal-free films deposited at a similar $\mathrm{P}_{\mathrm{Ar}}$ (see for example figure 1a.) This increased roughness may be due to the incorporation of metal droplets directly ablated from the target. As the pressure is increased the roughness of the samples increases, as was observed in the metal-free case. At $\mathrm{P}_{\mathrm{Ar}}=360 \mathrm{mTorr}$ (see figure $3 \mathrm{c}$ ) very lowdensity carbon NPC samples are observed, which appear again to have been assembled by diffusion limited aggregation. A similar morphology series with increasing $\mathrm{P}_{\mathrm{Ar}}$, was observed for films deposited using both graphite: metal mixed targets.

The nanostructure of the metal containing films was examined more closely in a TEM. Figure 4a shows a high resolution TEM of a NPC:Co sample deposited at $\mathrm{P}_{\mathrm{Ar}}=360 \mathrm{mTorr}$. The nanostructure of this sample appeared similar to that of the NPC sample deposited at the same pressure by ablating the pure graphite target. No metal nanoparticles were observed at the spatial resolution available, and selected area electron diffraction measurements showed no evidence for crystalline inclusions, however the amorphous matrix did appear to diffract more strongly than for the metal-free samples indicating the presence of heavier atoms. Any metal present is expected to be in or on the observed clusters.

The nanostructure of the NPC:Ni samples deposited by ablating the graphite:Ni mixed target was indistinguishable from the NPC:Co sample grown at the same $\mathrm{P}_{\mathrm{Ar}}$. However, differences were observed for the NPC:Ni samples deposited by ablating the graphite:Ni composite target. For this sample (see figure $4 \mathrm{~b}$ ) metal nanoparticles were detected, supported in the matrix of amorphous clusters. 


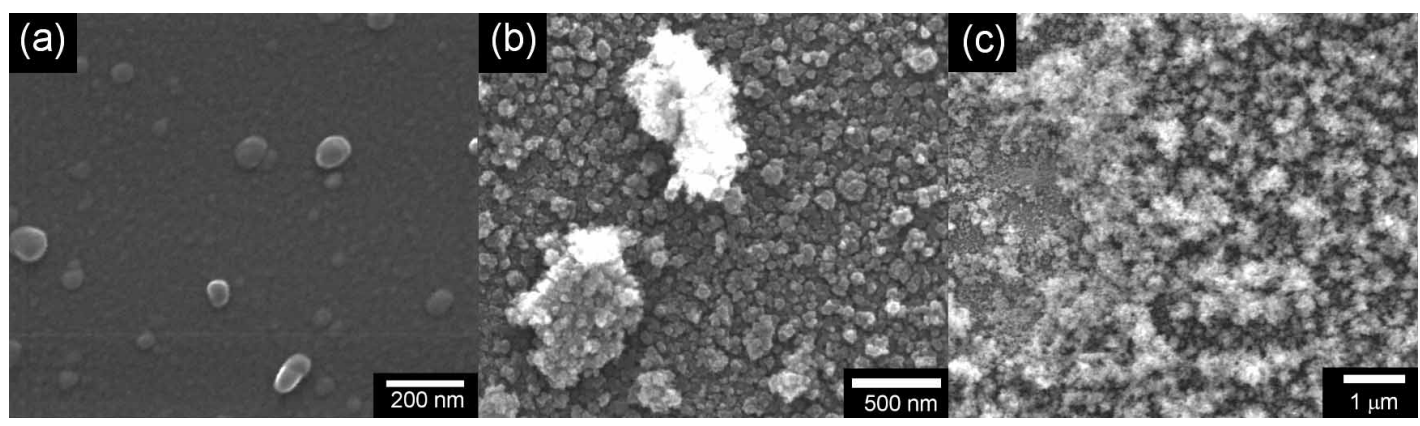

FIG. 3: SEM images of NPC:Ni samples, deposited by ablating the composite target, at different $\mathrm{P}_{\mathrm{Ar}}=$ (a) 0 mTorr, (b) 200 mTorr and (c) 360 mTorr.

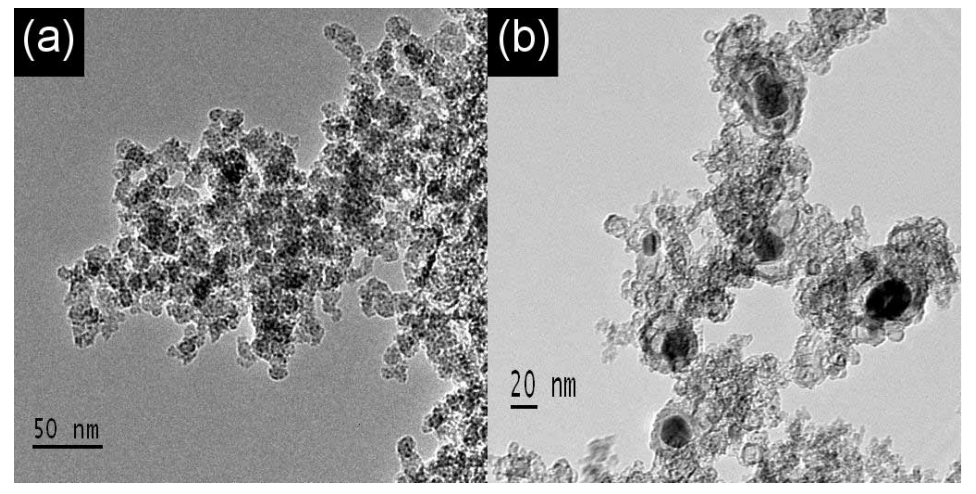

FIG. 4: TEM image of (a) NPC:Co and (b) NPC:Ni (composite target) deposited at $\mathrm{P}_{\mathrm{Ar}}=360$ mTorr.

There is also some evidence in figure $4 \mathrm{~b}$ for the presence of carbon shells around the metal nanoparticles. For the graphite: Ni composite target periodic ablation of the two sections of the target is performed. Thus, only the metal atoms/clusters that are resident in the chamber for times comparable with the period between ablating the different targets would have the opportunity to be included into a carbon cluster. Recent theoretical calculations [9] indicate that the diffusion time for carbon atoms and clusters in comparable background pressures, can be of the order of $1-10^{3}$ seconds, considerably longer than the successive material ablation period used here (around 0.5 seconds here.) However, the region for cluster formation will be limited to the high temperature shock front [9] which was observed, during the plume imaging experiments, to dissipate after a few microseconds after the laser pulse, so the periodic ablation would be expected to be less efficient at including metal atoms into carbon clusters.

In order to estimate the metal content of the samples EDX measurements were performed, at low accelerating voltages, on the films deposited on $\mathrm{Si}$. The data obtained was calibrated against samples with a known Ni:C ratio. The results of the EDX measurements are shown in figure 5. For both the NPC:Ni and the NPC:Co samples (see figures $5 \mathrm{a}$ and $\mathrm{b}$ ) the metal content was observed to decrease with increasing $\mathrm{P}_{\mathrm{Ar}}$. For the ablation in vacuum, the metal percentage was found to be higher (35-40\%) than that of both targets $(20 \%)$. This could be attributed to two mechanisms. Firstly, the evaporation of metal atoms from the target will occur more easily than for carbon as the melting point of the metals is much lower than the melting point of graphite. The second mechanism for the enhancement of the metal content involves the redeposition of the less volatile component back onto the target. This mechanism is discussed in more detail elsewhere [18]. At higher pressures the reduction in the metal content of the film is expected to be a consequence of the plume dynamics. At low pressures, the content of the film is expected to be very similar to that of the evaporated material. At intermediate pressures the mean free path of the larger metal atoms will be smaller than that of the carbon atoms, meaning that they will suffer more collisions, reducing the metal content of the films. At the higher pressures studied metal content appears relatively insensitive to further increase in the pressure, 

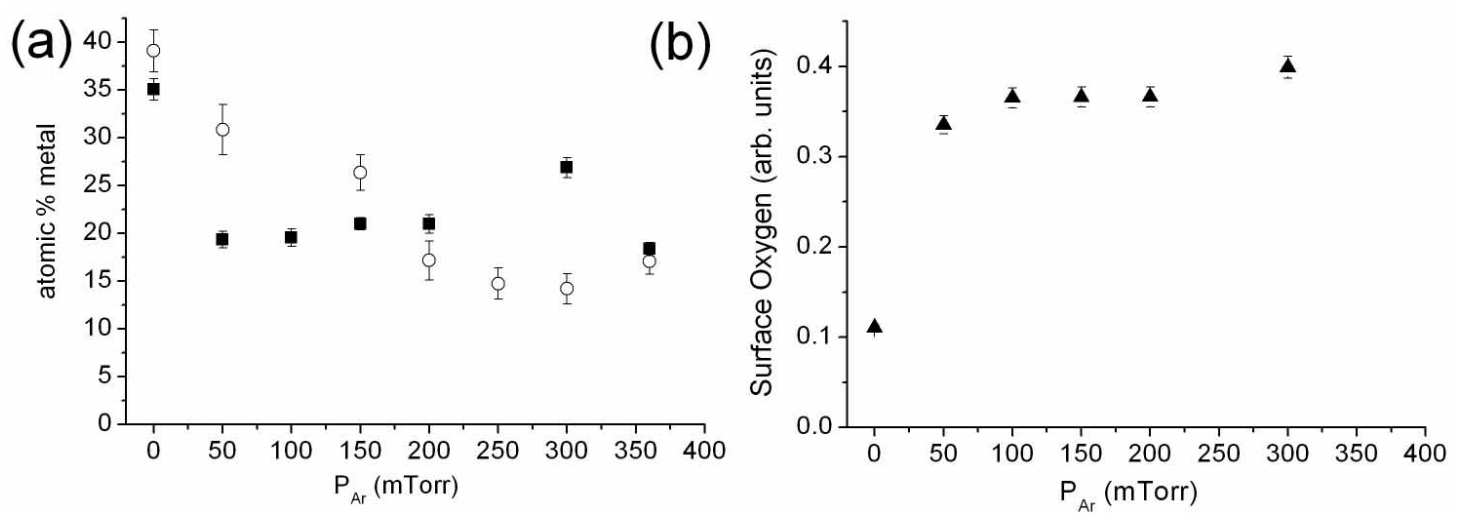

FIG 5. (a) Metal content for NPC:Co (-) and NPC:Ni (!) films deposited at different $\mathrm{P}_{\text {Ar. }}$ (b) Ratio of the O:C EDX signals for NPC:Ni films deposited at different $\mathrm{P}_{\mathrm{Ar}}$.

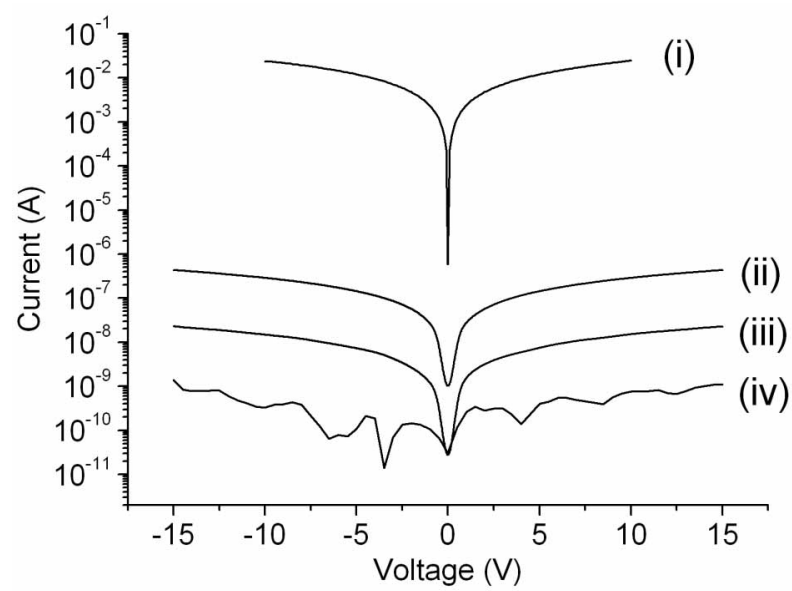

FIG. 6: IV data for NPC:Ni samples deposited at $\mathrm{P}_{\mathrm{Ar}}=$ (i) 0 mTorr, (ii) 100 mTorr, (iii) 200 mTorr, and (iv) 300 mTorr.

suggesting that the both species rapidly thermalise due to multiple collisions and that the metal content of the clusters is controlled by the relative propensities of the two species for clustering.

The larger surface area to volume ratio of the films deposited at higher $\mathrm{P}_{\mathrm{Ar}}$ is evidenced by the increased oxygen content of the films observed by EDX (see figure 5c). It is known that water and oxygen from the air can be chemisorbed as $\mathrm{COH}, \mathrm{C}=\mathrm{O}, \mathrm{COOH}$ or $\mathrm{C}-\mathrm{O}-\mathrm{C}$ groups on carbon [8], which could decrease the concentration of electrons near the surface and cause a depletion layer with a higher resistance. From the EDX measurements presented in figure 5 it can be observed that the oxygen to carbon EDX peak intensity ratio increased as $\mathrm{P}_{\mathrm{Ar}}$ was increased. This is attributed to the larger surface area to volume ratio of the rougher samples providing more sites for oxygen or water chemisorption / physisorption.

To investigate the effect of increasing roughness and increased surface oxygen on the resistivity of the metal containing NPC samples, the DC current-voltage (IV) characteristics of the films were measured. Figure 6 shows a series of IV plots for NPC:Ni samples, produced by ablating the mixed target, at different $\mathrm{P}_{\mathrm{Ar}}$. At $\mathrm{P}_{\mathrm{Ar}}$ $=0 \mathrm{mTorr}$ the films display Ohmic behavior and are conductive. As the pressure is increased the sheet resistance of the films rapidly increase. Above $\mathrm{P}_{\mathrm{Ar}}=300 \mathrm{mTorr}$ the samples were too resistive to accurately measure with the apparatus used. The most rapid rise in the sheet resistance of the NPC:Ni samples, with increasing $\mathrm{P}_{\mathrm{Ar}}$, occurred in the pressure range 0-100 mTorr. Over this same range, the oxygen to carbon ratio also rises most rapidly. However, in this range the increase in nano-scale clustering in the films will also increase the resistance, as percolation pathways will become much longer. 


\section{CONCLUSIONS}

The growth of nanoporous carbon materials with high metal content was investigated by pulsed laser ablation of metal-containing graphite targets. The roughness/porosity of the samples increased rapidly with increasing

$\mathrm{P}_{\mathrm{Ar}}$, and at the highest pressures studied the growth was characteristic of cluster assembly by diffusion-limited aggregation. The metal content and the sheet conductance of the samples were observed to decrease with increasing $\mathrm{P}_{\mathrm{Ar}}$. However, reasonable conductivity could be maintained in the samples, by the inclusion of metal up to background pressures of around 200-300 mTorr. The chemisorption / physisorption of water or oxygen onto the samples saturated above around $\mathrm{P}_{\mathrm{Ar}}=100$ mTorr indicating that samples deposited in this pressure range may still be useful for gas sensing applications, where a reasonable conductivity of the material is required, although the highest surface area to volume ratio was observed for the samples deposited at higher pressures.

\section{ACKNOWLEDGMENTS}

The authors are grateful to EPSRC for funding via portfolio and the Carbon Based Electronics programme. Plume imaging experiments were carried out at the Ultraviolet Laser Facility operating at IESL FORTH and supported by the EU through the Research Infrastructures activity of FP6 (Project:Laserlab-Europe contract No: RII3-CT-2003-506350). The authors thank D. Anglos and C. Fotakis for their assistance with this project.

\section{REFERENCES}

1. R. F. Wood, K. R. Chen, J. N. Leboeuf, A. A. Puretzky, and D. B. Geohegan, Phys. Rev. Lett. 79, 1571 (1997).

2. R. K. Thareja and R. K. Dwivedi, Phys. Lett. A 222, 199 (1996).

3. M. P. Siegal, D. L. Overmyer, R. J. Kottenstette, D. R. Tallant, and W. G. Yelton, Appl. Phys. Lett. 80, 3940 (2002).

4. A. A. Voevodin, S. J. P. Laube, S. D. Walck, J. S. Solomon, M. S. Donley, and J. S. Zabinski, J. Appl. Phys 78, 4123 (1995).

5. H. C. Ong and R. P. H. Chang, Phys. Rev. B 55, 13213 (1997).

6. Z. Geretovszky, T. Haraszti, T. Szörényi, F. Antoni, and E. Fogarassy, Appl. Surf. Sci. 208-209, 566 (2003).

7. B. S. Satyanarayana, J. Robertson, and W. I. Milne, J. Appl. Phys. 87, 3126 (2000).

8. M. Bruzzi, P. Piseri, S. Miglio, G. Bongiorno, E. Barborini, C. Ducati, J. Robertson, and P. Milani, Eur. Phys. J. B 36, 3 (2003).

9. A. V. Rode, E. G. Gamaly, and B. Luther-Davies, Appl. Phys. A 70, 135 (2000).

10. W. Wu and J. Ting, Thin Solid Films 420, 166 (2003).

11. H. Dimigen, H. Hübsch, and R. Memming, Appl. Phys. Lett. 50, 1056 (1987).

12. J. C. Orlianges, C. Champeaux, A. Catherinot, A. Pothier, P. Blondy, P. Abelard, and B. Angleraud, Thin Solid Films, 453, 291 (2004).

13. H. Wang, M. F. Chiah, W. Y. Cheung, and S. P. Wong, Phys. Lett. A 316, 122 (2003).

14. S. J. Henley, G. M. Fuge, and M. N. R. Ashfold, J. Appl. Phys. 97, 023304 (2005).

15. F. Claeyssens, M. N. R. Ashfold, E. Sofoulakis, C. G. Ristoscu, D. Anglos, and C. Fotakis, J. Appl. Phys. 91, 9 (2002).

16. A. A. Voevodin and M. S. Donley, Surf. Coatings Technol. 82, 199 (1996).

17. Y. B. Zel'dovich and Y. P. Raizer, Physics of Shock Waves and High-Temperature Hydrodynamic Phenomena (Academic Press, New York, 1966).

18. M. N. R. Ashfold, F. Claeyssens, G. Fuge, and S. J. Henley, Chem. Soc. Rev. 33, 23 (2004). 